\title{
International Meeting Molecular Neurodegeneration: News and Views in Molecular Neuroscience in Health and Disease. Delmenhorst, Germany, July 20-22, 2015
}

\author{
Illana Gozes ${ }^{1}$ - Peter W. Baas ${ }^{2}$ - Christiane Richter-Landsberg ${ }^{3}$
}

Published online: 29 August 2015

(C) Springer Science+Business Media New York 2015

\section{Organizers:}

Prof. Dr. Christiane Richter-Landsberg, Carl von Ossietzky Universität Oldenburg

Prof. Dr. Illana Gozes, Tel Aviv University

\section{Venue:}

Hanse-Wissenschaftskolleg (HWK)

Lehmkuhlenbusch 4

27753 Delmenhorst

\section{Illana Gozes}

igozes@post.tau.ac.il

Peter W. Baas

pbaas@drexelmed.edu

Christiane Richter-Landsberg

Christiane.Richter.Landsberg@uni-oldenburg.de

1 The Lily and Avraham Gildor Chair for the Investigation of Growth Factors, Elton Laboratory for Molecular Neuroendocrinology, Department of Human Molecular Genetics and Biochemistry, Sackler Faculty of Medicine, Sagol School of Neuroscience and Adams Super Center for Brain Studies, Tel Aviv University, Tel Aviv 69978, Israel

2 Department of Neurobiology and Anatomy, College of Medicine, Drexel University, 2900 Queen Lane, Philadelphia, PA 19129, USA

3 Department of Neuroscience, Molecular Neurobiology, University of Oldenburg, D-26111 Oldenburg, Germany

\section{Overview}

With the advances in medicine and extended longevity, neurodegenerative diseases become more prevalent and present major unmet medical needs with suffering to the patients, their families, and society as a whole. The aim of the HWK Molecular Neurodegeneration Symposium was to bring together experts on the molecular basis of neurodegeneration, paving the path to the identification of new molecular targets for early diagnosis, prevention, and intervention. The venue presented an excellent site to promote formal and informal exchange of ideas that will lead to further collaborations and new findings. Neuroscience students from surrounding universities presented posters and took part in the discussions, opening new horizons to the next generation scientists and promoting research in Lower Saxony, Germany, and worldwide. A special lecture was devoted to industry connections, trying to translate basic findings to practical drug candidates.

Topics

1. Molecular aspects in neurodegeneration

2. The cytoskeleton and associated proteins

3. Microtubules and brain disease

4. Glial cells and disease 


\section{Extended Meeting Report}

Molecular aspects in neurodegeneration were addressed from several angles, one included looking at nerve growth factor (NGF) and showing that a failure in NGF precursor maturation in Down's syndrome brains and a likely enhanced proteolytic degradation of NGF, may compromise the trophic support of basal forebrain cholinergic neurons. Thus, proNGF and NGF degrading enzymes pave the path to novel therapeutic targets in chronic neurodegenerative condition leading to dementia (Iulita et al. 2014).

On the other side of the spectrum, producing dimeric peptide-based inhibitors of postsynaptic density-95 (PSD95) and showing that they can reduce ischemic brain damage in rodents that are ready for further clinical development were discussed. Additional approaches were discussed, such as modifying the pharmacokinetic profile by designing a series of fatty acid linked dimeric ligands in a manner that potently inhibits PSD-95 and thereby improves in vitro blood plasma stability. Subcutaneous administration in rats demonstrated stability and sustained release of these ligands. These findings facilitate new pharmacological uses of PSD-95 inhibitors and further exploration of PSD-95 as a drug target for acute nerve injury such as in stroke (Nissen et al. 2015), in specialized stroke ambulance (http:/www.sciencedaily.com/releases/ 2014/04/140422162305.htm). It was refreshing to hear an advocate for peptide-related drug development, who first screened thousands of small molecules and reverted to very potent newly designed peptides for further clinical development. In this respect and speaking of peptides, an interesting neuroprotective mechanism was suggested for ghrelin, a stomach secreted peptide that protects dopaminergic neuron upon activation by oxygen, or rather oxygenated water, a simple neuroprotective mechanism (Matsumoto et al. 2013).

Tying chronic neurodegeneration with acute injury, tauoapthy and Wallerian degeneration were discussed, opening a new window to common and disparate mechanisms (Garland et al. 2012).

Looking at sensory organs such as the eyes and the ears, with degeneration affecting a large proportion of the population, it became obvious that understanding basic mechanisms is of crucial importance. Here, a focus was on the guanylate cyclase-activating proteins (GCAPs) that when mutated can cause blindness. Understanding the precise biochemical/biophysical interactions of these molecules will no doubt lead to further developments for treatments for devastating blindness (Sulmann et al. 2015).

At the center stage of the meeting was the cytoskeleton, spanning from basic aspects to disease and drug development. The cytoskeleton, with emphasis on microtubules, is essential for brain cell structure and function. The microtubules are composed of assembled tubulin backbone and decorated by microtubuleassociated proteins. Tubulin is the major brain protein and neurodegeneration is profoundly impacted by cytoskeletal breakdown. In Alzheimer's disease and related neurodegenerations, the microtubule associated protein tau (tubulin associated unit) forms pathological aggregates, termed neurofibrillary tangles. Microtubules and tau are major targets for drug development and there is currently no neuroprotective drug on the market that targets them. The conference assessed new findings at the basic aspects, also exploring further horizons, with world experts. Importantly, microtubule-associated proteins are deregulated in Alzheimer's disease and frontotemporal dementias affecting cognition, schizophrenia (mental illnesses), and Parkinson disease as well as frontotemporal dementias (motor dysfunctions); the conference was aimed at understanding similarities and differences between cytoskeletal degeneration in these versatile diseases toward better diagnosis and disease management. A major cellular mechanism that depends on intact, functioning microtubules is called autophagy, self-cleaning, a process by which the cell digests misfolded proteins to yield building blocks (starting materials) for properly folded proteins. The association between autophagy, microtubules, diagnosis, and drug development was elaborated (RichterLandsberg and Leyk 2013, Esteves et al. 2014, Merenlender-Wagner et al. 2014, Gozes 2015, Merenlender-Wagner et al. 2015).

Historically, there has been broad agreement among basic scientists that microtubules are essential for the complex morphology of the neuron, the establishment and maintenance of distinct functional compartments of the neuron, and for the directional transport of cargoes within axons and dendrites. However, there has been resistance to the idea that microtubule abnormalities are at the heart of neurodegenerative disorders such as tauopathies, including Alzheimer's disease and related dementias, Parkinson's disease, and others. That attitude 
is now changing, as the community learns that so many of the proteins and pathways that go awry in such disorders are microtubule-based (Cappelletti et al. 2015). Moreover, loss of microtubules or changes in the stability, severing or composition of microtubules is now recognized as a hallmark of axonal and dendritic degeneration. In the past, it has been argued that such changes are late effects in disease progression and hence not relevant to early diagnosis or treatment. Newer thinking is that the microtubule changes are ongoing long before the disease symptoms are apparent, but only become histologically dramatic in the late phases of disease. Preventing or even correcting microtubule abnormalities will not cure a neurodegenerative disorder, but it would likely help stave off the neural degeneration that degrades the quality of life for the patient.

There was robust discussion during the meeting about what exactly happens to the microtubules during neurodegeneration, which is at the heart of what should be done to treat the diseases. There was a discussion on how different diseases might affect microtubule stability, namely whether it is the more stable or labile microtubule fraction that is being primarily lost, and also discussion on the tubulin post-translational modifications that accompany microtubule stability and specialize different classes of microtubules to interact with different proteins. An interesting unexpected finding showed that microtubule-associated protein 6 , tightly linked to schizophrenia, mediates neuronal connectivity through Semaphorin 3Edependent signaling for axonal growth (Deloulme et al. 2015), two unexpected partners, perhaps paving the path to further similar interactions.

There was a discussion on new methods for discerning the levels of free tubulin relative to microtubules in the axons of cultured neuronal cells (Igaev et al. 2014), as well as discussion on how a straightforward drug approach could be used to quantify the levels of stable and labile microtubule mass.

Microtubule-severing proteins were discussed in light of diseases that might render microtubule abnormally sensitive to spastin (one of the microtubule-severing proteins), by abnormally increasing microtubule polyglutamylation (Magiera and Janke 2013). It was also proposed that pathogenic mutations of spastin may not cause hereditary spastic paraplegia by diminishing microtubule severing but rather by changing microtubule dynamics via gain-of-function effects of the mutant protein (Solowska and Baas 2015). Manipulating particular severing proteins was also suggested as a potential therapy for tauopathy and other diseases because knockdown of particular severing proteins can alter the levels of labile versus stable microtubule mass.

Finally, there was much discussion on activity-dependent neuroprotective protein (ADNP) (Bassan et al. 1999, Gozes et al. 2015), with mutations to ADNP causing autism associated with cognitive dysfunction (Helsmoortel et al. 2014, Gozes et al. 2015). ADNP, a chromatin remodeling factor that has the cytoplasmic role of interacting with a class of proteins called microtubule end-binding proteins that track the plus ends of microtubules (Oz et al. 2014). EBs recognize a nucleotide-dependent structural cap, while tracking the growing microtubule ends (Maurer et al. 2012).

An amino acid snippet of ADNP called NAP enhances the interaction of the full-length protein with microtubule end-binding proteins (Oz et al. 2014). Thus, NAP could serve as a powerful therapeutic alternative to microtubule-stabilizing drugs that may or may not fortify levels of labile microtubule mass when used at low concentrations.

In short, microtubules, major building blocks of the cytoskeleton, were discussed as targets for drug design in versatile neurodegenerative and neuropsychiatric diseases, and NAP is currently under clinical development.

A second focus of the meeting is glial cells and neurodegenerative diseases. While neurodegenerative disease focus on the degeneration of neurons, it is important to acknowledge that glial cells (the brain support cells, which constitute a larger part of the brain) play an important role in neuroprotection and neurodegeneration. As such, microglia display a dual role, while those cells may be linked to the potential clearance of aggregated proteins, such as the amyloid plaques, these cells are associated with neuroinflammation and support brain tumor growth (Szulzewsky et al. 2015).

Oligodendrocytes were highlighted with connection to exosomes. As previously reviewed, information exchange executed by extracellular vesicles, including exosomes, is a newly described form of intercellular communication important in the development and physiology of neural systems. These vesicles can be released from cells, are packed with information including signaling proteins and both coding and regulatory RNAs, 
and can be taken up by target cells, thereby facilitating the transfer of multilevel information. Exosomes have a critical role in physiological processes, including nerve regeneration, axonal transport, synaptic function, and behavior. These vesicles also have a sinister role in the propagation of toxic amyloid proteins in neurodegenerative conditions, including prion diseases and Alzheimer's and Parkinson's diseases, in inducing neuroinflammation by exchange of information between the neurons and glia, as well as in aiding tumor progression in the brain by subversion of normal cells (Rajendran et al. 2014). Oligodendrocyte-derived exosomes situated between the myelin sheath and the axon may affect axonal properties and axonal transport. Oligodendrocytes, the myelin forming cells of the CNS, are characterized by their numerous membranous extensions, which enwrap neuronal axons and form myelin sheaths. During differentiation, oligodendrocytes pass different morphological stages, downregulate the expression of the proteoglycan NG2, and acquire major myelin specific proteins, such as myelin basic proteins (MBP) and proteolipid protein. The meeting organizers, Christiane Richter-Landsberg and Illana Gozes, were the first to identify tubulin, the subunit of microtubules in myelin (Gozes and Richter-Landsberg 1978). Figure 1 shows the microtubular network in an oligodendroglial cell, as performed in the Richter-Landsberg laboratory. It

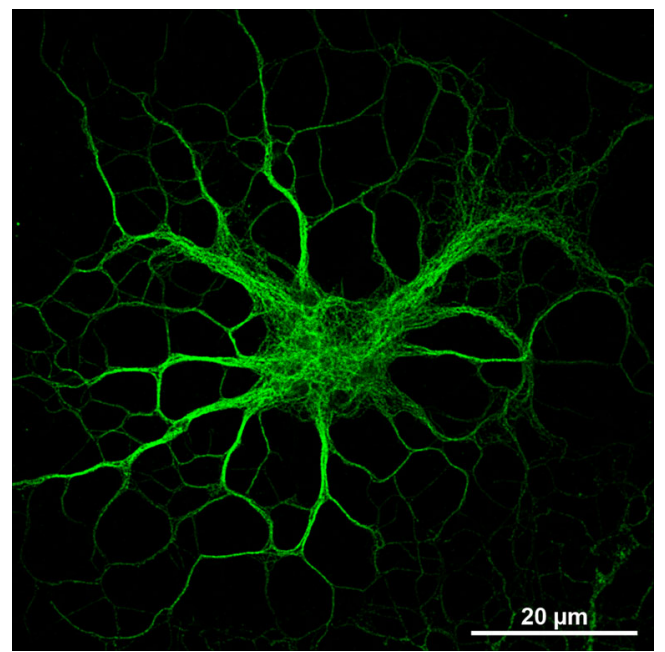

Fig. 1 Microtubules in an oligodendrocyte (for experimental methods please, see Seiberlich et al. 2015) has later been show that MBP mRNA is transported in RNA granules along the microtubules to the periphery and translated locally. The microtubule-associated protein (MAP), tau, is present in oligodendrocytes and involved in the regulation and stabilization of the microtubule network. Tau knockdown impairs process outgrowth and leads to a decrease in MBP expression. Furthermore, MBP mRNA transport to distant cellular extensions is impaired and cells remain in the NG2 stage. In myelinating co-cultures with dorsal root ganglion neurons, oligodendrocyte precursor cell tau knockdown results NG2-positive cells. Thus, tau is important for MBP mRNA transport and is involved in process formation. The disturbance of the balance of tau leads to abnormalities in oligodendrocyte differentiation, neuron-glia contact formation and the early myelination process (Seiberlich et al. 2015). The intricate neuro-glia interaction is dependent on intact molecular machineries including the cytoskeleton and the autophagy process and comparisons were made to further knowledge and translate into better disease management.

The meeting was timely, inspiring ideas to form consortia toward Horizon 2020 and putting excellent German science in close contact with European and world leaders toward better solutions for devastating, prevalent brain disease. Putting the cytoskeleton, and neuro-glia interactions at a center point, will allow for future better insights, as well as diagnostics and drug development. The meeting included scientists from Europe, Germany, France, Italy, the UK, Belgium, Spain and Denmark, the Middle East, Israel, Asia, Japan and North America, the USA, and Canada. The proportion between senior scientists and young investigators was balanced, with 15 exceptional posters and, out of which $\sim 50 \%$ were chosen for oral presentations and 1 received an award, supported by the HWK.

While estimates of prevalence vary, few would dispute the multitude of patients and the urgent need to find ways to prevent, delay, and treat Alzheimer's disease, frontotemporal dementias, Parkinson's, and other motor disorders as well as mental diseases. Understanding the core of the diseases and focus on central regulatory elements within the nerve cell and its support cells is a road not so much travelled and this is our focus toward new horizons (see Table 1 for the complete meeting program). 
Table 1 Meeting program

Monday July 20, 2015

09:20-09:30

09:30-10:10

10:10-12:40

10:10-10:50

11:20-12:00

12:00-12:40

15:50 - $16: 40$

15:50 - 16:10

$16: 10-16: 40$

$16: 40-17: 10$

17:10-17:50

Tuesday July 21, 2015

09:00-12:10

09:00-09:40

09:40-10:20

10:50-11:30

11:30-12:10

15:30-18:00

15:30-16:10

16:10-16:50

17:20-18:00

Wednesday July 22, 2015

09:30-10:20

09:30-10:20

10:20-10:45

10:45-11:00

11:00-11:15

11:15-11:30

11:30-11:45

11:45-12:00

12:00 - 12:10
Welcome address

Special lecture, Chair: Illana Gozes (Tel Aviv, Israel)

Claudio Cuello (Montreal, Canada): A nerve growth factor dysmetabolism in Alzheimer's and in Down Syndrome

Session 1: Glial cells and disease, Chair: Christiane Richter-Landsberg (Oldenburg, Germany)

Helmut Kettenmann (Berlin, Germany): Role of microglia in pathology

Mami Noda (Fukuoka, Japan): Microglial dysfunction and neuronal damage in neurodegeneration.

Eva-Maria Krämer-Albers (Mainz, Germany): The role of exosomes in neuron-glia communication and neuroprotection

Session 2: Cytoskeleton and beyond, Chair: Annie Andrieux (Grenoble, France)

Poster talk 1: Amrit Mudher (UK) Protection of axonal degeneration in a transgenic model of Tauopathy

Poster talk 2: Frank Kooy (Belgium) A SWI/SNF-related autism syndrome caused by de novo mutation in ADNP

Special lecture, Chair: Ulrike Janssen-Bienhold (Oldenburg, Germany)

Karl Koch (Oldenburg, Germany): Molecular mechanisms of retinal degeneration

Session 3: Microtubules and brain disease, Chair: Claudio Cuello (Montreal, Canada) and Marie-Francoise Suaud-Chagny (Bron, France)

Peter Baas (Philadelphia, USA): Microtubules: Loss-of-function and gain-of-function mechanisms contributing to neurodegeneration

Illana Gozes (Tel Aviv, Israel): Cytoskeleton as drug target for neuroprotection

Annie Andrieux (Grenoble, France): Microtubule dysfunctions in neuro -degenerative -psychiatric diseases

Graziela Cappelletti (Milan, Italy): Looking at microtubule dysfunction in toxin- and gene-based models of Parkinson's disease

SESSION 4: Molecular aspects in neurodegeneration: the cytoskeleton and associated proteins, Chair: Peter Baas (Philadelphia, USA)

Sebastian Maurer (Barcelona, Spain): Microtubule plus end tracking and dynamic instability are mechanistically linked

Magdalena Magiera (Paris, France): Microtubule posttranslational modifications

Christiane Richter-Landsberg (Oldenburg, Germany): Protein aggregate formation in oligodendrocytes: The cytoskeleton and autophagy at the intersection of neuroprotection and neurodegeneration.

Session 5: Mechanisms to clinical trials, Chair: Markus Kipp (Munich, Germany)

Kristian Stromgard (Copenhagen, Denmark): Translational medicine and drug design

Poster talks, Chair: Ephraim Yavin (Rehovot, Israel)

Poster talk 3, Benedict Niewidok (Osnabruk, Germany): Quantitative live cell imaging of microtubule dynamics in living neuronal cells

Poster talk 4, Janina Leyk (Oldenburg, Germany): Inhibition of HDAC6 results in altered cellular responses to proteasomal and oxidative stress in retinal cells

Poster talk 5, Felix Bulcke (Bremen, Germany): Copper oxide nanoparticles: Synthesis, toxic potential and modulation of astrocytic metabolism

Poster talk 6, Farina Vocke (Oldenburg, Germany): Dysfunction of cGMP signaling in photoreceptors by a Macular Dystrophy related mutation in the calcium sensor GCAP1

Poster talk 7, Tina Schlüter (Oldeburg, Germany): Role of microRNA-96 for development and function of the auditory brainstem

Poster prize: S. Sulmann (Oldenburg, Germany) Fingerprint profile of cone dystrophy related GCAP1 mutants 
Table 1 (continued)

Poster-only presentations:

A similar aberrant regulation of retinal guanylate cyclase by two missense mutations in GUCA1A associated with retinal dystrophy arises from distinct molecular mechanisms

Valerio Marino, Alexander Scholten, Karl-Wilhelm Koch, Daniele Dell’Orco (Oldeburg, Germany)

The microtubule associated protein tau is involved in oligodendroglial process formation and early myelination processes in cultured oligodendrocytes Veronika Seiberlich, Nina G Bauer, Lisa Schwarz, Charles ffrench-Constant, Olaf Goldbaum, Christiane Richter-Landsberg (Oldenburg, Germany) Radixin regulates synaptic GABAA receptor density and is essential for reversal learning and short-term memory

Torben J. Hausrat, Mary Muhia, Kimberly Gerrow, Philip Thomas, Wiebke Hirdes, Sachiko Tsukita, Frank F. Heisler, Lena Herich, Sylvain Dubroqua, Petra Breiden, Joram Feldon, Jürgen R. Schwarz, Benjamin K. Yee, Trevor G. Smart, Antoine Triller and Matthias Kneussel (Presenter--Hamburg,

Germany)

Oligodendrocyte damage triggers immune-cell recruitment into the forebrain

Markus Kipp (Munich, Germany)

MOAG-4/SERF: a direct modifier of amyloid assembly in neurodegenerative diseases

N. Helge Meyer, Tobias Madl, Klaus Zangger, Fabio Falsone (Presenter: Oldenburg, Germany)

Uptake and effects of iron oxide nanoparticles in primary cultures of microglia, astrocytes and neurons

Charlotte Petters, Karsten Thiel, Ralf Dringen (Bremen, Germany)

Biological effects of repeated electroconvulsive seizures (ECS) in MAP6-KO mice, an animal model of psychiatric disorders

Marie-Françoise Suaud-Chagny, Julie Jonckheere, Gaëlle Dall' Igna, Jean-Christophe Deloulme, Nicolas Chauliac, Christophe Porcher, Jérôme

Holtzmann, Annie Andrieux, Sylvie Gory-Fauré (Presneter-Lyon, France)

Acknowledgments The meeting was supported by the Deutsche Forschungsgemeinschaft (Ri 384/18-1), the Institute for Advanced Study Hanse-Wissenschaftskolleg (HWK), Delmenhorst, Germany and Springer-Nature Press (the Journal of Molecular Neuroscience). Professor Illana Gozes is a Humboldt Award Recipient, and both Professors Gozes and Baas were fellows at HWK. Professor Richter-Landsberg serves on the Scientific Advisory Board of HWK. We thank Dr. Dorothe Poggel and the HWK staff for their significant contribution to the meeting organization.

\section{References}

Bassan M, Zamostiano R, Davidson A, Pinhasov A, Giladi E, Perl O, Bassan H, Blat C, Gibney G, Glazner G, Brenneman DE, Gozes I (1999) Complete sequence of a novel protein containing a femtomolar-activity-dependent neuroprotective peptide. J Neurochem 72:1283-1293

Cappelletti G, Casagrande F, Calogero A, De Gregorio C, Pezzoli G, Cartelli D (2015) Linking microtubules to Parkinson's disease: the case of parkin. Biochem Soc Trans 43:292-296

Deloulme JC, Gory-Faure S, Mauconduit F, Chauvet S, Jonckheere J, Boulan B, Mire E, Xue J, Jany M, Maucler C, Deparis AA, Montigon O, Daoust A, Barbier EL, Bosc C, Deglon N, Brocard J, Denarier E, Le Brun I, Pernet-Gallay K, Vilgrain I, Robinson PJ, Lahrech H, Mann F, Andrieux A (2015) Microtubule-associated protein 6 mediates neuronal connectivity through semaphorin 3Edependent signalling for axonal growth. Nat Commun 6:7246

Esteves AR, Gozes I, Cardoso SM (2014) The rescue of microtubuledependent traffic recovers mitochondrial function in Parkinson's disease. Biochim Biophys Acta 1842:7-21

Garland P, Broom LJ, Quraishe S, Dalton PD, Skipp P, Newman TA, Perry VH (2012) Soluble axoplasm enriched from injured CNS axons reveals the early modulation of the actin cytoskeleton. PLoS One 7:e47552.

Gozes I (2015) The cytoskeleton as a drug target for neuroprotection: the case of the autism- mutated ADNP. Biol Chem
Gozes I, Helsmoortel C, Vandeweyer G, Van der Aa N, Kooy F, Sermone SB (2015) The compassionate side of neuroscience: tony sermone's undiagnosed genetic journey-ADNP mutation. J Mol Neurosci 56: 751-757

Gozes I, Richter-Landsberg C (1978) Identification of tubulin associated with rat brain myelin. FEBS Lett 95:169-172

Helsmoortel C, Vulto-van Silfhout AT, Coe BP, Vandeweyer G, Rooms L, van den Ende J, Schuurs-Hoeijmakers JH, Marcelis CL, Willemsen MH, Vissers LE, Yntema HG, Bakshi M, Wilson M, Witherspoon KT, Malmgren H, Nordgren A, Anneren G, Fichera M, Bosco P, Romano C, de Vries BB, Kleefstra T, Kooy RF, Eichler EE, Van der Aa N (2014) A SWI/SNF-related autism syndrome caused by de novo mutations in ADNP. Nat Genet 46:380-384

Igaev M, Janning D, Sundermann F, Niewidok B, Brandt R, Junge W (2014) A refined reaction-diffusion model of tau-microtubule dynamics and its application in FDAP analysis. Biophys J 107:25672578

Iulita MF, Do Carmo S, Ower AK, Fortress AM, Aguilar LF, Hanna M, Wisniewski T, Granholm AC, Buhusi M, Busciglio J, Cuello AC (2014) Nerve growth factor metabolic dysfunction in Down's syndrome brains. Brain 137:860-872

Magiera MM, Janke C (2013) Investigating tubulin posttranslational modifications with specific antibodies. Methods Cell Biol 115: 247-267

Matsumoto A, Yamafuji M, Tachibana T, Nakabeppu Y, Noda M, Nakaya H (2013) Oral 'hydrogen water' induces neuroprotective ghrelin secretion in mice. Sci Rep 3:3273

Maurer SP, Fourniol FJ, Bohner G, Moores CA, Surrey T (2012) EBs recognize a nucleotide-dependent structural cap at growing microtubule ends. Cell 149:371-382

Merenlender-Wagner A, Malishkevich A, Shemer Z, Udawela M, Gibbons A, Scarr E, Dean B, Levine J, Agam G, Gozes I (2015) Autophagy has a key role in the pathophysiology of schizophrenia. Mol Psychiatry 20:126-132

Merenlender-Wagner A, Shemer Z, Touloumi O, Lagoudaki R, Giladi E, Andrieux A, Grigoriadis NC, Gozes I (2014) New horizons in schizophrenia treatment: autophagy protection is coupled with behavioral improvements in a mouse model of schizophrenia. Autophagy 10:2324-2332 
Nissen KB, Andersen JJ, Haugaard-Kedstrom LM, Bach A, Stromgaard K (2015) Design, synthesis, and characterization of fatty acid derivatives of a dimeric peptide-based postsynaptic density-95 (PSD-95) inhibitor. J Med Chem 58:1575-1580

Oz S, Kapitansky O, Ivashco-Pachima Y, Malishkevich A, Giladi E, Skalka N, Rosin-Arbesfeld R, Mittelman L, Segev O, Hirsch JA, Gozes I (2014) The NAP motif of activity-dependent neuroprotective protein (ADNP) regulates dendritic spines through microtubule end binding proteins. Mol Psychiatry 19:1115-1124

Rajendran L, Bali J, Barr MM, Court FA, Kramer-Albers EM, Picou F, Raposo G, van der Vos KE, van Niel G, Wang J, Breakefield XO (2014) Emerging roles of extracellular vesicles in the nervous system. J Neurosci 34:15482-15489

Richter-Landsberg C, Leyk J (2013) Inclusion body formation, macroautophagy, and the role of HDAC6 in neurodegeneration. Acta Neuropathol 126:793-807
Seiberlich V, Bauer NG, Schwarz L, Ffrench-Constant C, Goldbaum O, Richter-Landsberg C (2015) Downregulation of the microtubule associated protein tau impairs process outgrowth and myelin basic protein mRNA transport in oligodendrocytes. Glia 63:1621-1635

Solowska JM, Baas PW (2015) Hereditary spastic paraplegia SPG4: what is known and not known about the disease. Brain

Sulmann S, Vocke F, Scholten A, Koch KW (2015) Retina specific GCAPs in zebrafish acquire functional selectivity in $\mathrm{Ca}(2+)$-sensing by myristoylation and $\mathrm{Mg}(2+)$-binding. Sci Rep 5:11228

Szulzewsky F, Pelz A, Feng X, Synowitz M, Markovic D, Langmann T, Holtman IR, Wang X, Eggen BJ, Boddeke HW, Hambardzumyan D, Wolf SA, Kettenmann H (2015) Glioma-associated microglia/ macrophages display an expression profile different from M1 and M2 polarization and highly express Gpnmb and Spp1. PLoS One 10:e0116644. 